\title{
On the unitarity of gauged non-compact world-sheet supersymmetric WZNW models
}

\author{
Jonas Björnsson 1 and Stephen Hwang 2 \\ Department of Physics \\ Karlstad University \\ SE-651 88 Karlstad, Sweden
}

\begin{abstract}
In this paper we generalize our investigation of the unitarity of non-compact WZNW models connected to Hermitian symmetric spaces to the $\mathrm{N}=1$ world-sheet supersymmetric extension of these models. We will prove that these models have a unitary spectrum in a BRST approach for antidominant highest weight representations if the level and weights of the gauged subalgebra are integers. We will find new critical string theories in 7 and 9 space-time dimensions.
\end{abstract}

\section{Introduction}

In a previous paper [1] we considered strings on group manifolds connected to Hermitian symmetric spaces of non-compact type. These are constructed by starting with a noncompact group $G$ which has maximal compact subgroup $H$ with a one-dimensional center $Z(H)$. Then, the coset space $G / H$ is a Hermitian symmetric space of non-compact type. All these spaces have been classified. They have $G$ being $S U(p, q), S O(p, 2), S O^{*}(2 n)$ and $S p(2 p, \mathbb{R})$. Furthermore, there are two connected to exceptional groups, $E_{6}\left(E_{6 \mid-14}\right)$ and $E_{7}\left(E_{7 \mid-25}\right)$. As the maximal compact subgroup has a one-dimensional center, one can use these groups to construct non-trivial space-time backgrounds where strings can propagate. Write the maximal compact subgroup as $H=H^{\prime} \times Z(H)$ and construct the coset $G / H^{\prime}$.

This background has a compact direction which plays the rôle of time. One can formulate this as a WZNW model, as was done in [2] and prove it to be unitary using a BRST approach [1. If one assumes that the weights and level of the subalgebra are

\footnotetext{
${ }^{1}$ jonas.bjornsson@kau.se

${ }^{2}$ stephen.hwang@kau.se
} 
integer\$3, the spectrum is unitary. This is not the case for the Goddard-Kent-Olive coset construction [1]. In a realistic string model one would take the infinite cover so that the time is uncompactified. Furthermore, we do not consider sectors corresponding to spectral flow, which was originally proposed in [3] and further elaborated in refs. [4]-6] for the $S L(2, \mathbb{R})$ string. Such sectors would be crucial in constructing an S-matrix unitary string theory, as modular transformations mix different spectrally flowed sectors for the $S L(2, \mathbb{R})$ string.

In this paper we will extend our analysis and consider the $\mathcal{N}=1$ world-sheet supersymmetric extension of the WZNW model 77 8]. This model has bosonic and fermionic degrees of freedom. These two sectors mix in a trivial way and can be decoupled by redefining the affine generators. Constructing coset models of these theories was first done in [9] for the bosonic case and a simple $\mathcal{N}=1$ supersymmetric extension of WZNW model. The generalization to other world-sheet supersymmetric WZNW models was considered in [10] [11] and is, therefore, called the Kazama-Suzuki coset construction. We will in this paper use a BRST approach to the coset construction [12], as this formulation gives unitarity for the bosonic case [1. The BRST formulation of the Kazama-Suzuki model was considered in [13] and [14].

The relevant representations which we consider here are so-called antidominant highest weight representations. In addition, we will assume that the level and weights of the corresponding subalgebra are integers. We will find that the $\mathcal{N}=1$ world-sheet supersymmetric extension of the WZNW-model has a unitary spectrum. At the end of the paper we will give a table of the relevant coset models. Some of these models will by themselves yield criticality. These occur in seven dimensions: $S U(3,1) / S U(3)$ at level $k=-6$ and $S O(3,2) / S O(3)$ at level $k=-6$; in nine dimensions: $S U(4,1) / S U(4)$ at level $k=-40$ and $S O(4,2) / S O(4)$ at level $k=-32$.

In our previous paper [1, we also considered the necessity of our assumed representations. The proof given there is, however, not in general complete. It holds when the rank of $\mathfrak{g}$ is two (see below for explanation of notation). We will in a forthcoming publication discuss more general cases.

Let us introduce some notations and basic definitions. We will in general follow the conventions and notations used in [15]. Denote by $\mathfrak{g}$ and $\mathfrak{h}$ the Lie algebras corresponding to the non-compact group $G$ and its maximal compact subgroup $H$, which admit a Hermitian symmetric space of the form $G / H$. Let $\mathfrak{g}^{\mathbb{C}}$ and $\mathfrak{h}^{\mathbb{C}}$ denote the corresponding complex Lie algebras. We will always take the rank $r_{\mathfrak{g}}$ of $\mathfrak{g}$ to be greater than one and $\mathfrak{g}$ to be simple. One knows that $\mathfrak{h}$ has a one-dimensional center, thus one can split $\mathfrak{h}$ as $\mathfrak{h}^{\prime} \oplus \mathfrak{u}(1)$. We choose $\mathfrak{h}^{\mathbb{C}}$ such that it is a regular embedding in $\mathfrak{g}^{\mathbb{C}}$ i.e. using the Cartan-Weyl basis, the Cartan elements of $\mathfrak{h}^{\mathbb{C}}$, as well as generators corresponding to positive/negative roots,

\footnotetext{
${ }^{3}$ The level of the algebra $\hat{\mathfrak{g}}$ is integer, or if the Dynkin index is different from one, also half integer.
} 
are all in the corresponding decomposition of $\mathfrak{g}^{\mathbb{C}}$.

Denote by $\Delta$ all roots, $\Delta^{+/-}$the positive/negative roots, $\Delta_{s}$ the simple roots, $\Delta_{c}$ the compact roots, $\Delta_{c}^{+}=\Delta_{c} \cap \Delta^{+}$the compact positive roots, $\Delta_{n}$ the non-compact roots and $\Delta_{n}^{+}$the positive non-compact roots. We take the long roots to have length $\sqrt{2}$. Let $\alpha \in \Delta$ and define the coroot by $\alpha^{\vee}=2(\alpha, \alpha)^{-1} \alpha$. Let $\alpha^{(i)} \in \Delta^{+}$denote the simple roots. When we need to distinguish between different root systems, we denote by $\Delta^{\mathfrak{g}}$ and $\Delta^{\mathfrak{h}^{\prime}}$ the roots in $\mathfrak{g}^{\mathbb{C}}$ and $\mathfrak{h}^{\prime \mathbb{C}}$, respectively. Furthermore, we use capital letters, $A, B, \ldots$, and small letter, $a, b, \ldots$, to denote elements in the algebra $\mathfrak{g}$ and $\mathfrak{h}^{\prime}$ respectively. Denote by $J^{A}$ a generic generator of $\hat{\mathfrak{g}}^{\mathbb{C}}$ which in a Cartan-Weyl basis is

$$
\begin{aligned}
J^{i} & =H^{i}, \quad i=1, \ldots, r_{\mathfrak{g}} ; \\
J^{\alpha} & =E^{\alpha}, \quad \alpha \in \Delta .
\end{aligned}
$$

We fix the basis of the root space such that the highest root is non-compact. In addition, one can choose the basis of roots such that if $\alpha \in \Delta_{c}^{\mathfrak{g}}$ then the first component is zero and the other $r_{\mathfrak{g}}-1$ components are, in general, non-zero. This, furthermore, yields an isomorphism between $\Delta_{c}^{\mathfrak{g}}$ and $\Delta^{\mathfrak{h}^{\prime}} . g_{\mathfrak{g}}^{\vee}$ denotes the dual Coxeter number of $\mathfrak{g}$. It is wellknown that one can choose a basis such that there is a unique non-compact simple root and if $\alpha \in \Delta_{n}^{+}$then the coefficient of the non-compact simple root is always one in a simple root decomposition of $\alpha$. Dynkin diagrams and relations between positive non-compact roots of the Lie-algebras are presented in the appendix of [16] and, with our notation, in [1]. For the embedding of $\mathfrak{h}^{\prime}$ in $\mathfrak{g}$, one defines the Dynkin index of an embedding

$$
I_{\mathfrak{h}^{\prime} \subset \mathfrak{g}}=\frac{(\theta(\mathfrak{g}), \theta(\mathfrak{g}))}{\left(\theta\left(\mathfrak{h}^{\prime}\right), \theta\left(\mathfrak{h}^{\prime}\right)\right)},
$$

where, for regular embeddings, $\theta\left(\mathfrak{h}^{\prime}\right)$ is the highest root of $\mathfrak{h}^{\prime}$ in $\mathfrak{g}$. The conformal anomaly for the $G / H^{\prime}$ WZNW model is

$$
c_{\text {tot }}=\frac{\left(k-g_{\mathfrak{g}}^{\vee}\right) \operatorname{dim}(\mathfrak{g})}{k}+\frac{1}{2} \operatorname{dim}(\mathfrak{g})-\frac{\left(\kappa-g_{\mathfrak{h}^{\prime}}^{\vee}\right) \operatorname{dim}\left(\mathfrak{h}^{\prime}\right)}{\kappa}-\frac{1}{2} \operatorname{dim}\left(\mathfrak{h}^{\prime}\right),
$$

where $\kappa$ is defined as

$$
\kappa \equiv I_{\mathfrak{h}^{\prime} \subset \mathfrak{g}} k .
$$

Let us use operator product language to write down the relations for the algebra. The operator product expansions (OPE's) of the $\mathcal{N}=1$ superaffine algebra are

$$
\begin{array}{ll}
J^{A}(z) J^{B}(w) & \sim k \kappa^{A B}(z-w)^{-2}+f_{C}^{A B} J^{C}(w)(z-w)^{-1} \\
J^{A}(z) \lambda^{B}(w) & \sim f^{A B}{ }_{C} \lambda^{C}(w)(z-w)^{-1} \\
\lambda^{A}(z) \lambda^{B}(w) & \sim k \kappa^{A B}(z-w)^{-1} \times\left\{\begin{array}{ll}
1 & \text { for R - fermions } \\
\frac{1}{2}\left(\sqrt{\frac{z}{w}}+\sqrt{\frac{w}{z}}\right) & \text { for NS - fermions }
\end{array},\right.
\end{array}
$$


where $\sim$ means equality up to regular terms, $f{ }^{A B}{ }_{C}$ and $\kappa^{A B}$ are the structure constants and killing form, respectively, for the corresponding finite dimensional Lie algebra. The non-zero values in a Cartan-Weyl basis of $\mathfrak{g}$ are

$$
\begin{aligned}
f_{\beta}^{i \alpha} & =\alpha^{i} \delta_{\alpha, \beta} \\
f_{i}^{\alpha \beta} & =\alpha_{i}^{\vee} \delta_{\alpha+\beta, 0} \\
f_{\gamma}^{\alpha \beta} & =e_{\alpha, \beta} \delta_{\alpha+\beta, \gamma} \\
\kappa^{i j} & =G^{i j} \\
\kappa^{\alpha \beta} & =\frac{2}{(\alpha, \alpha)} \delta_{\alpha+\beta, 0},
\end{aligned}
$$

where $G^{i j} \equiv\left(\alpha^{(i)^{\vee}}, \alpha^{(j)^{\vee}}\right)$ and $e_{\alpha, \beta}$ is non-zero iff $\alpha+\beta$ is a root. The correspondence between OPE's and brackets aret

$$
\left[A_{m}, B_{n}\right]=\frac{1}{(2 \pi \mathrm{i})^{2}} \oint d w w^{\Delta_{B}+n-1} \oint d z z^{\Delta_{A}+m-1} \mathcal{R}(A(z) B(w)),
$$

where $\Delta$ is the conformal dimension of the operator and $\mathcal{R}$ is the radial ordering of the operators

$$
\mathcal{R}(A(z) B(w))=\left\{\begin{array}{rr}
A(z) B(w) & |z|>|w| \\
(-)^{\epsilon_{A} \epsilon_{B}} B(w) A(z) & |z|<|w|
\end{array}\right.
$$

where $\epsilon$ denote the Grassman parity of the operator. The radial ordering satisfies

$$
\mathcal{R}(A(z) B(w)) \sim: A(w) B(w):+A(z) B(w),
$$

where : : means normal ordering. The element corresponding to the center, $\hat{\mathfrak{u}}(1)$, is

$$
H_{m}=\Lambda_{(1) i} H_{m}^{i}
$$

where $\Lambda_{(i)}$ are fundamental weights which are defined by $\left(\Lambda_{(i)}, \alpha^{(j)^{\vee}}\right)=\delta_{i}^{j} . H_{m}$ satisfies

$$
\begin{aligned}
& {\left[H_{m}, E_{n}^{ \pm \alpha}\right]=0, \quad \alpha \in \Delta_{c}^{+}} \\
& {\left[H_{m}, E_{n}^{ \pm \beta}\right]= \pm E_{m+n}^{ \pm \beta}, \quad \beta \in \Delta_{n}^{+} .}
\end{aligned}
$$

One can also define new currents

$$
\hat{J}^{A}(z)=J^{A}(z)+\frac{1}{2 k} f_{B C}^{A}: \lambda^{B} \lambda^{C}(z):
$$

which make the OPE's between $\lambda^{A}$ and $\hat{J}^{A}$ consist of only regular terms. Furthermore, these currents satisfy an affine algebra of level $k-g^{\vee}$.

\footnotetext{
${ }^{4}$ This is for bosonic operators and Ramond fermions.
} 


\section{Coset construction, the BRST approach}

The Kazama-Suzuki coset construction is defined by considering states $|\Phi\rangle$ in a representation of the supersymmetric affine algebra and then gauging a subalgebra by the conditions

$$
J_{+}^{\hat{\mathfrak{h}}^{\prime}}|\Phi\rangle=\lambda_{+}^{\hat{\mathfrak{h}}^{\prime}}|\Phi\rangle=0
$$

where $J_{+}^{\hat{h}^{\prime}}$ and $\lambda_{+}^{\hat{\mathfrak{h}}^{\prime}}$ are generic annihilation operators in the subalgebra $\hat{\mathfrak{h}}^{\prime}$. In the BRST approach one introduces instead a BRST operator to define the coset space [13] [14]. One needs to introduce, in addition to the ghost sector, an auxiliary sector with currents $\tilde{J}^{a}(z)$ for the subalgebra $\hat{\mathfrak{h}}^{\prime}$. The BRST current is 5

$$
\begin{aligned}
j_{B R S T}^{1} & =c_{a}\left(\hat{J}^{a}-\frac{1}{2 k} f_{B C}^{a}{ }_{B} \lambda^{B} \lambda^{C}+\hat{\tilde{J}}^{a}-\frac{1}{2 \kappa} f_{b c}^{a} \tilde{\lambda}^{b} \tilde{\lambda}^{c}\right)+\gamma_{a}\left(\lambda^{a}+\tilde{\lambda}^{a}\right) \\
& -\frac{1}{2} f^{a b}{ }_{c} c_{a} c_{b} b^{c}+f^{a b}{ }_{c} c_{a} \gamma_{b} \beta^{c},
\end{aligned}
$$

where $c_{a}$ and $\gamma_{a}$ are fermionic and bosonic ghosts, respectively, with corresponding momenta $b^{a}$ and $\beta^{a}$. They satisfy

$$
\begin{aligned}
c_{a}(z) b^{b}(w) & \sim \delta_{a}^{b}(z-w)^{-1} \\
\gamma_{a}(z) \beta^{b}(w) & \sim \delta_{a}^{b}(z-w)^{-1} .
\end{aligned}
$$

The OPE of the BRST current defined in eq. (2.2) with itself yields only regular terms if $\kappa+\tilde{\kappa}=0$. Thus, we take the level of the extra sector $\tilde{\kappa}$ to be $-\kappa$. To get the physical subspace we need to add contributions to this BRST charge arising from the superconformal symmetry. One finds

$$
\begin{aligned}
j_{B R S T} & =: c_{a}\left(\hat{J}^{a}-\frac{1}{2 k} f_{B C}^{a} \lambda^{B} \lambda^{C}+\hat{\tilde{J}}^{a}-\frac{1}{2 \kappa} f_{b c}^{a} \tilde{\lambda}^{b} \tilde{\lambda}^{c}\right):+: \gamma_{a}\left(\lambda^{a}+\tilde{\lambda}^{a}\right): \\
& +: c T:+: \gamma G:-\frac{1}{2} f^{a b}{ }_{c}: c_{a} c_{b} b^{c}:+f^{a b}{ }_{c}: c_{a} \gamma_{b} \beta^{c}: \\
& -: \partial c c b:-: \gamma^{2} b+: \partial c \gamma \beta:+\frac{1}{2}: c \gamma \partial \beta:-\frac{1}{2}: c \partial \gamma \beta: \\
& +: c \partial b^{a} c_{a}:+\frac{3}{2}: c \partial \beta^{a} \gamma_{a}:+\frac{1}{2}: c \beta^{a} \partial \gamma_{a}:+: \partial c b^{a} c_{a}: \\
& +: \partial c \beta^{a} \gamma_{a}:-: \gamma b^{a} \gamma_{a}:-: \gamma \partial \beta^{a} c_{a}:-: \partial \gamma \beta^{a} c_{a}:,
\end{aligned}
$$

\footnotetext{
${ }^{5}$ Here we have assumed that $\mathfrak{h}^{\prime}$ is simple. If it is not one has for each simple part the BRST current as in eq. (2.2). Henceforth, we will present equations which hold for the case when $\mathfrak{h}^{\prime}$ is simple as the generalization to the semisimple case is straightforward.

${ }^{6}$ In a mode expansion, $\gamma_{a, m}$ is chosen to have hermiticity properties $\gamma_{i, m}^{\dagger}=\gamma_{i, m}, \gamma_{\alpha, m}^{\dagger}=\gamma_{-\alpha, m}$, $\beta_{i, m}^{\dagger}=-\beta_{i, m}$ and $\beta_{\alpha, m}^{\dagger}=-\beta_{-\alpha, m}$.
} 
where

$$
\begin{aligned}
T(z) & =\frac{1}{2 k} \kappa_{A B}\left(: \hat{J}^{A} \hat{J}^{B}:+: \partial \lambda^{A} \lambda^{B}:\right) \\
& +\frac{1}{2 \tilde{\kappa}} \kappa_{a b}\left(: \hat{\tilde{J}}^{a} \hat{\tilde{J}}^{b}:+: \partial \tilde{\lambda}^{a} \tilde{\lambda}^{b}:\right)+T^{\prime}(z) \\
G(z) & =\frac{1}{k}\left(\kappa_{A B}: \hat{J}^{A} \lambda^{B}(z):-\frac{1}{6 k} f_{A B C}: \lambda^{A} \lambda^{B} \lambda^{C}(z):\right) \\
& +\frac{1}{\tilde{\kappa}}\left(\kappa_{a b}: \hat{\tilde{J}}^{a} \tilde{\lambda}^{b}(z):-\frac{1}{6 \tilde{\kappa}} f_{a b c}: \tilde{\lambda}^{a} \tilde{\lambda}^{b} \tilde{\lambda}^{c}(z):\right)+G^{\prime}(z),
\end{aligned}
$$

where $T^{\prime}(z)$ and $G^{\prime}(z)$ originates from a unitary superconformal algebra making the conformal anomaly equal to 15 as the corresponding BRST charge is only nilpolent for $c=15$.

Furthermore, we have in the BRST charge introduced superconformal ghosts and corresponding momenta. These have the non-zero OPE's

$$
\begin{aligned}
& c(z) b(w) \sim(z-w)^{-1} \\
& \gamma(z) \beta(w) \sim(z-w)^{-1} .
\end{aligned}
$$

From the BRST current one can define a few useful charges, one is the zero mode for the Virasoro algebra,

$$
\begin{aligned}
L_{0}^{\mathrm{tot}} & =\left[Q, b_{0}\right] \\
& =L_{0}+\sum_{m \in \mathbb{Z}}\left(m: b_{-m} c_{m}:+m: b_{-m}^{a} c_{m, a}:\right) \\
& +\sum_{r \in \mathbb{Z}+\nu}\left(r: \beta_{-r} \gamma_{r}:+r: \beta_{-r}^{a} \gamma_{r, a}:\right)+a_{g},
\end{aligned}
$$

and the other corresponds to the Cartan subalgebra of the Lie algebra

$$
\begin{aligned}
H_{0}^{\mathrm{tot}, i} & =\left[Q, b_{0}^{i}\right] \\
& =\hat{H}_{0}^{i}+\hat{\tilde{H}}_{0}^{i}-\sum_{m, \alpha \in \Delta} \frac{(\alpha, \alpha)}{4 k} \alpha^{i}: \lambda_{-m}^{-\alpha} \lambda_{m}^{\alpha}:-\sum_{m, \alpha \in \Delta_{c}} \frac{(\alpha, \alpha)}{4 \tilde{\kappa}} \alpha^{i}: \tilde{\lambda}_{-m}^{-\alpha} \tilde{\lambda}_{m}^{\alpha}: \\
& +\sum_{m, \alpha \in \Delta_{c}} \alpha^{i}: b_{-n}^{\alpha} c_{n, \alpha}:+\sum_{r \in \mathbb{Z}+\nu, \alpha \in \Delta_{c}} \alpha^{i}: \beta_{-r}^{\alpha} \gamma_{r, \alpha}:,
\end{aligned}
$$

where $a_{g}=-1 / 2$ or $a_{g}=0$ is a normal ordering constant and $\nu=0$ or $\nu=1 / 2$ for the Neveu-Schwarz and Ramond sectors, respectively. 


\section{The statespace}

We consider here only discrete highest weight representations of the non-compact real forms. To be more precise, we consider the representations which have a highest weight that is antidominant. This subclass of highest weights will be defined below. The representations are constructed by using redefined currents where the world-sheet fermions and affine currents decouple. Furthermore, we work in the complex algebra and choose a specific hermitian conjugation of the operators to get representations of the relevant real form. A hermitian conjugation rule which yields the correct representations is

$$
\begin{aligned}
\left(\hat{E}_{-n}^{\alpha}\right)^{\dagger} & =-\hat{E}_{n}^{-\alpha} \\
\left(\lambda_{-n}^{\alpha}\right)^{\dagger} & =-\lambda_{n}^{-\alpha}
\end{aligned}
$$

where $\alpha \in \Delta_{n}$. The rest of the operators satisfy

$$
\left(A_{-n}\right)^{\dagger}=A_{n} .
$$

The highest weight state in both the Neveu-Schwarz and Ramond sectors is of the form

$$
|0 ; \mu, \tilde{\mu}\rangle \equiv|0 ; \mu\rangle \otimes|0 ; \tilde{\mu}\rangle \otimes|0\rangle_{\text {ghost }} .
$$

For the Neveu-Schwarz sector, we have

$$
A_{m}|0 ; \mu, \tilde{\mu}\rangle=0 \text {, }
$$

for $m>0$, where $A$ denotes a generic operator. For $m=0$ one has

$$
\begin{aligned}
A_{0}^{\alpha}|0 ; \mu\rangle & =0 \\
\hat{H}_{0}^{i}|0 ; \mu\rangle & =\mu^{i}|0 ; \mu\rangle, \\
b_{0}^{i}|0\rangle_{\text {ghost }}=b_{0}|0\rangle_{\text {ghost }} & =0
\end{aligned}
$$

where $\alpha \in \Delta_{+}, A=\{E, c, b\}$ and $i=1, \ldots, r$. We have here defined

$$
c_{m}^{\alpha}=\frac{(\alpha, \alpha)}{2} \kappa^{\alpha \beta} c_{m, \beta}=c_{m,-\alpha}
$$

and similary for the bosonic ghosts.

For the highest weight state in the Ramond sector the difference from the NeveuSchwarz case comes from the zero modes for the world-sheet fermions and bosonic ghosts. The representations are constructed in the same way as for the bosonic generators with the 
exception of the fermions corresponding to the Cartan subalgebra. For these we introduce creation and annihilation operators by linear combinations

$$
\lambda_{0}^{\tilde{a}, \pm}= \begin{cases}\frac{1}{\sqrt{2}}\left( \pm \lambda_{0}^{\prime 2 \tilde{a}-1}+\mathrm{i} \lambda_{0}^{\prime 2 \tilde{a}}\right) & \mathrm{r}_{\mathfrak{g}}=2 \mathbb{Z} \\ \frac{1}{\sqrt{2}}\left( \pm \lambda_{0}^{\prime 2 \tilde{a}}+\mathrm{i} \lambda_{0}^{\prime 2 \tilde{a}+1}\right) & \mathrm{r}_{\mathfrak{g}}=2 \mathbb{Z}+1\end{cases}
$$

where $\tilde{a}=1, \ldots,\left[r_{\mathfrak{g}} / 2\right] 7$. Here, $\lambda_{0}^{\prime i}$ are linear combinations of $\frac{1}{\sqrt{-k}} \lambda_{0}^{j}$ such that

$$
\left[\lambda_{0}^{\prime i}, \lambda_{0}^{\prime j}\right]=-\delta^{i, j}
$$

From these operators one can construct a set of states by defining a highest weight state that satisfies

$$
\lambda_{0}^{\tilde{a},+}|0 ; \mu\rangle=\lambda_{0}^{\alpha}|0 ; \mu\rangle=0 .
$$

This construction also generalizes to the bosonic ghosts,

$$
\gamma_{0}^{\alpha}|0\rangle_{\text {ghost }}=\beta_{0}^{\alpha}|0\rangle_{\text {ghost }}=\gamma_{0}^{\tilde{a},+}|0\rangle_{\text {ghost }}=\beta_{0}^{\tilde{a},+}|0\rangle_{\text {ghost }}=0
$$

where $\gamma_{0}^{\tilde{a},+}$ and $\beta_{0}^{\tilde{a},+}$ are constructed as in eq. (3.7), $\alpha \in \Delta_{c}^{+}$and $\tilde{a}=1, \ldots,\left[r_{\mathfrak{h}^{\prime}} / 2\right]$. For $r_{\mathfrak{g}}$ odd, there is one extra component of $\lambda^{\prime i}$ which is left out of the above construction. This gives an extra two-fold degeneration, which is of no importance to us and will be disregarded in the following.

The highest weight state satisfies

$$
\begin{aligned}
H_{0}^{\mathrm{tot}, i}|0 ; \mu, \tilde{\mu}\rangle & =\left(\mu^{i}+\tilde{\mu}^{i}+h_{g}^{i}\right)|0 ; \mu, \tilde{\mu}\rangle \\
L_{0}^{\mathrm{tot}}|0 ; \mu, \tilde{\mu}\rangle & =\left(\frac{\mathcal{C}_{2}^{\mathfrak{g}}}{2 k}+\frac{\mathcal{C}_{2}^{\mathfrak{h}^{\prime}}}{2 \tilde{\kappa}}+a_{g}\right)|0 ; \mu, \tilde{\mu}\rangle,
\end{aligned}
$$

where $a_{g}$ is $-1 / 2$ or 0 and $h_{g}^{i}$ is $2 \rho_{\hat{\mathfrak{h}}}^{i}$ or $\rho_{\mathfrak{g}}^{i}+\rho_{\mathfrak{h}^{\prime}}^{i}$ for the Neveu-Schwarz and the Ramond sectors, respectively 8 . The states are now constructed by applying the redefined creation operators on the highest weight state. As the fermions and ghosts describe a free theory the corresponding statespaces are simple. For the redefined currents the representations are unchanged, except that the level is shifted to $k-g_{\mathfrak{g}}^{\vee}$ and $\tilde{\kappa}-g_{\mathfrak{h}}^{\vee}$ for the $\hat{\mathfrak{g}}$ - and $\hat{\tilde{\mathfrak{h}}}$-sector,

\footnotetext{
${ }^{7}[$ ] denotes the integer part.

${ }^{8}$ The contribution to $h_{g}^{i}$ has different sources in the different sectors, for the Neveu-Schwarz sector it arises from the fermionic ghosts and from the Ramond sector it arises from the world-sheet fermions.
} 
respectively. Thus, unitary representations with a highest weight $\hat{\tilde{\mu}}$, called dominant integral, satisfy for the $\hat{\tilde{\mathfrak{h}}}^{\prime}$-sector

$$
\begin{aligned}
\tilde{\mu}^{i} & \geq 0 \quad i=2, \ldots, r_{\mathfrak{g}} \\
\tilde{\kappa} & \geq g_{\mathfrak{h}^{\prime}}^{\vee}+\left(\theta_{\mathfrak{h}^{\prime}}, \mu\right) .
\end{aligned}
$$

and antidominant representations for the $\hat{\mathfrak{g}}$-sector are representations, with a highest weight $\hat{\mu}$ that satisfies

$$
\begin{aligned}
\mu^{i} & <-\rho^{i} \quad i=1, \ldots, r_{\mathfrak{g}} \\
k & <\left(\theta_{\mathfrak{g}}, \mu\right) .
\end{aligned}
$$

The Verma modules corresponding to antidominant weights are irreducible, as can be deduced from the Shapovalov-Kac-Kazhdan determinant [17] [18.

For well-known reasons, one does not consider the cohomology of the corresponding BRST charge of the current defined in eq. (2.4), but the relative cohomology defined by the conditions

$$
\begin{aligned}
Q|\Phi\rangle & =0 & & \text { NS and R sectors } \\
b_{0}^{i}|\Phi\rangle & =0 & & \text { NS and R sectors } \\
b_{0}|\Phi\rangle & =0 & & \text { NS and R sectors } \\
\beta_{0}|\Phi\rangle & =0, & & \text { R sector and } r_{\mathfrak{g}} \in 2 \mathbb{Z}+1
\end{aligned}
$$

where $i=2, \ldots, r_{g}$.

\section{Unitarity}

The mass shell condition is of the form

$$
\left(\frac{\left(\mu, \mu+2 \rho_{\mathfrak{g}}\right)}{2 k}+\frac{\left(\tilde{\mu}, \tilde{\mu}+2 \rho_{\mathfrak{h}^{\prime}}\right)}{2 \tilde{\kappa}}\right)+N+l^{\prime}+a_{g}=0
$$

where $l^{\prime} \geq 0$ originates from some unitary SCFT. Following [1, define

$$
\chi^{\left(\hat{g}, \hat{h}^{\prime} \oplus S V i r\right)}(\tau, \phi, \theta) \equiv \operatorname{Tr}\left[\exp \left[2 \pi \mathrm{i} \tau\left(L_{0}^{\mathrm{tot}}\right)\right] \exp \left[\mathrm{i} \sum_{i=2}^{r_{\mathfrak{g}}} \theta_{i} H_{0}^{\mathrm{tot}, i}+\mathrm{i} \phi H_{0}\right](-1)^{\Delta N_{g h}}\right]
$$

$\Delta N_{g h}$ is the ghostnumber of the state relative to the highest weight state for the ghosts. The trace is taken over all states in $\mathcal{H}_{\hat{\mu}}^{\hat{g}} \times \mathcal{H}_{\tilde{\tilde{\mu}}}^{\hat{\tilde{h}}^{\prime}} \times \mathcal{H}_{l^{\prime}}^{S C F T} \times \mathcal{H}^{\prime g h o s t} . \mathcal{H}_{\ldots}^{A}$ denotes the statespace of $A$. Therefore, the character can be decomposed into separate parts

$$
\chi^{\left(\hat{g}, \hat{h}^{\prime} \oplus S V i r\right)}(\tau, \phi, \theta)=e^{2 \pi \mathrm{i} \tau a_{\mathfrak{g}}} \chi^{1}(\tau, \phi, \theta) \chi^{\hat{\tilde{h}}^{\prime}}(\tau, \theta),
$$


where we have defined

$$
\chi^{1}(\tau, \phi, \theta) \equiv \chi^{\hat{g}}(\tau, \phi, \theta) \chi^{S C F T}(\tau) \chi^{g h o s t}(\tau, \theta) .
$$

As is well known [19], the character defined in eq. (4.2) only gets contributions from nontrivial BRST invariant states. However, since we are interested in the relative cohomology, where the eigenvalues of $L_{0}^{\text {tot }}$ and $H_{0}^{\text {tot, } i}$ are zero, one needs to project onto such states. We need, therefore, to consider the function

$$
\int d \tau \mathcal{B}^{\left(\hat{g}, \hat{h}^{\prime} \oplus S V i r\right)}(\tau, \phi) \equiv \int d \tau \int \prod_{i=2}^{r_{\mathfrak{g}}}\left(d \theta_{i}\right)\left\{\chi^{\left(\hat{g}, \hat{h}^{\prime} \oplus S V i r\right)}(\tau, \phi, \theta)\right\} .
$$

The $\tau$ - and $\theta_{i}$-integrations are formal integrations which project onto the $\tau$ - and $\theta_{i}$ independent part, making the character compatible with eq. (3.14). $\left.\mathcal{B}^{\left(\hat{g}, \hat{\bar{h}}^{\prime} \oplus \text { SVir }\right.}\right)(\tau, \phi)$ is called the generalized branching function. This definition was first introduced in [20] and extended in [21]. As in [1, define another function, the signature function

$$
\begin{aligned}
\Sigma^{\left(\hat{\mathfrak{g}}, \hat{\mathfrak{h}}^{\prime} \oplus \text { SVir }\right)}(\tau, \phi, \theta) & \\
& \equiv \operatorname{Tr}^{\prime}\left[\exp \left[2 \pi \mathrm{i} \tau\left(L_{0}^{\mathrm{tot}}\right)\right] \exp \left[\mathrm{i} \sum_{i=2}^{r_{\mathfrak{g}}} \theta_{i} H_{0}^{\mathrm{tot}, i}+\mathrm{i} \phi H_{0}\right](-1)^{\Delta N_{g h}}\right],
\end{aligned}
$$

The prime on the trace indicates that the trace is taken with signs i.e. a state with positive (negative) norm contributes with a positive (negative) sign in the trace. The corresponding coset signature function is defined as

$$
\int d \tau \mathcal{S}^{\left(\hat{\mathfrak{g}}, \hat{\mathfrak{h}}^{\prime} \oplus S V i r\right)}(\tau, \phi) \equiv \int d \tau \int \prod_{i=2}^{r_{\mathfrak{g}}}\left(d \theta_{i}\right)\left\{\Sigma^{\left(\hat{\mathfrak{g}}, \hat{\mathfrak{h}}^{\prime} \oplus S V i r\right)}(\tau, \phi, \theta)\right\} .
$$

The relevance of these functions to the present problem was stated is (cf. Lemma 2 in [1]).

Lemma $1 \mathcal{H}_{\hat{\mu} \tilde{\mu}}^{Q}$ is unitary if, and only if,

$$
\int d \tau\left[\mathcal{B}^{\left(\hat{\mathfrak{g}}, \hat{\mathfrak{h}}^{\prime} \oplus S V i r\right)}(\tau, \phi)-\mathcal{S}^{\left(\hat{\mathfrak{g}}, \hat{\mathfrak{h}}^{\prime} \oplus S V i r\right)}(\tau, \phi)\right]=0
$$

Here $\mathcal{H}_{\hat{\mu} \tilde{\mu}}^{Q}$ denotes the irreducible subspace of $\mathcal{H}_{\hat{\mu}}^{\hat{g}} \times \mathcal{H}_{\hat{\tilde{\mu}}}^{\hat{h}^{\prime}} \times \mathcal{H}_{l^{\prime}}^{S C F T} \times \mathcal{H}^{\prime g h o s t}$ consisting of non-trivial states in the relative cohomology.

We can now state our main result. 
Theorem 1 Let $\hat{\mu}$ be an antidominant weight. If $\hat{\tilde{\mu}}$ is a dominant integral weight then $\mathcal{H}_{\hat{\mu} \hat{\mu}}^{Q}$ is unitary.

Note that due to eq. (3.12), the components of $\hat{\mu}$ which belong to the subalgebra need to be integers since otherwise $\mathcal{H}_{\hat{\mu} \hat{\mu}}^{Q}$ is trivial.

Proof: Let us prove this theorem using Lemma 1. We need to determine the character in each sector. As the character decomposes into separate parts, much is already known from [1]. We need to derive the fermionic part of the $\hat{\mathfrak{g}}$-sector and the contribution corresponding to the bosonic ghosts. Let us first consider the NS-sector. In this sector the part of the character in $\hat{\mathfrak{g}}$ which is due to the fermions is

$$
\begin{aligned}
\chi_{\mu}^{\mathfrak{g} \text { Part. }}(q, \theta, \phi) & =\prod_{m=1}^{\infty}\left[\left(1+q^{m-1 / 2}\right)^{r_{\mathfrak{g}}} \prod_{\alpha \in \Delta_{c}}\left(1+q^{m-1 / 2} \exp [\mathrm{i}(\alpha, \theta)]\right)\right. \\
& \times \prod_{\alpha \in \Delta_{n}^{+}}\left(1+q^{m-1 / 2} \exp [\mathrm{i} \phi] \exp \left[\mathrm{i}\left(\alpha_{\|}, \theta\right)\right]\right) \\
& \left.\times \prod_{\alpha \in \Delta_{n}^{+}}\left(1+q^{m-1 / 2} \exp [-\mathrm{i} \phi] \exp \left[-\mathrm{i}\left(\alpha_{\|}, \theta\right)\right]\right)\right]
\end{aligned}
$$

where we have defined $q=\exp [2 \mathrm{i} \pi \tau]$ and $\alpha_{\|} \equiv \sum_{i=2}^{r_{\mathfrak{g}}} \alpha^{i} \Lambda_{(i)}$. As the compact roots have no first component, we suppress the $\|$ notation for these roots. Together with the bosonic contribution, see [1], the character for the $\hat{\mathfrak{g}}$-sector is

$$
\begin{aligned}
\chi_{\mu}^{\mathfrak{g}}(q, \theta, \phi) & =q^{\frac{\mathcal{g}^{\mathfrak{g}}(\mu)}{2 k}} \exp [\mathrm{i}(\mu, \Theta)] \prod_{\alpha \in \Delta_{c}^{+}} \frac{1}{1-\exp [-\mathrm{i}(\alpha, \theta)]} \\
& \times \prod_{\alpha \in \Delta_{n}^{+}} \frac{1}{1-\exp [-\mathrm{i} \phi] \exp \left[-\mathrm{i}\left(\alpha_{\|}, \theta\right)\right]} \\
& \times \prod_{m=1}^{\infty}\left[\left(\frac{1+q^{m-1 / 2}}{1-q^{m}}\right)^{r_{\mathfrak{g}}} \prod_{\alpha \in \Delta_{c}} \frac{1+q^{m-1 / 2} \exp [\mathrm{i}(\alpha, \theta)]}{1-q^{m} \exp [\mathrm{i}(\alpha, \theta)]}\right. \\
& \times \prod_{\alpha \in \Delta_{n}^{+}} \frac{1+q^{m-1 / 2} \exp [\mathrm{i} \phi] \exp \left[\mathrm{i}\left(\alpha_{\|}, \theta\right)\right]}{1-q^{m} \exp [\mathrm{i} \phi] \exp \left[\mathrm{i}\left(\alpha_{\|}, \theta\right)\right]} \\
& \left.\times \prod_{\alpha \in \Delta_{n}^{+}} \frac{1+q^{m-1 / 2} \exp [-\mathrm{i} \phi] \exp \left[-\mathrm{i}\left(\alpha_{\|}, \theta\right)\right]}{1-q^{m} \exp [-\mathrm{i} \phi] \exp \left[-\mathrm{i}\left(\alpha_{\|}, \theta\right)\right]}\right],
\end{aligned}
$$


where we have defined $(\mu, \Theta)=2\left(\alpha^{(1)}, \alpha^{(1)}\right)^{-1} \mu_{1} \phi+\sum_{i=2}^{r_{\mathfrak{g}}} \mu_{i} \theta^{i}$. The signature function is determined in the same way. One finds

$$
\begin{aligned}
\Sigma_{\mu}^{\mathfrak{g}}(q, \theta, \phi) & =q^{\frac{\mathcal{C}_{2}^{\mathfrak{g}}(\mu)}{2 k}} \exp [\mathrm{i}(\mu, \Theta)] \prod_{\alpha \in \Delta_{c}^{+}} \frac{1}{1+\exp [-\mathrm{i}(\alpha, \theta)]} \\
& \times \prod_{\alpha \in \Delta_{n}^{+}} \frac{1}{1-\exp [-\mathrm{i} \phi] \exp \left[-\mathrm{i}\left(\alpha_{\|}, \theta\right)\right]} \\
& \times \prod_{m=1}^{\infty}\left[\left(\frac{1-q^{m-1 / 2}}{1+q^{m}}\right)^{r_{\mathfrak{g}}} \prod_{\alpha \in \Delta_{c}} \frac{1-q^{m-1 / 2} \exp [\mathrm{i}(\alpha, \theta)]}{1+q^{m} \exp [\mathrm{i}(\alpha, \theta)]}\right. \\
& \times \prod_{\alpha \in \Delta_{n}^{+}} \frac{1+q^{m-1 / 2} \exp [\mathrm{i} \phi] \exp \left[\mathrm{i}\left(\alpha_{\|}, \theta\right)\right]}{1-q^{m} \exp [\mathrm{i} \phi] \exp \left[\mathrm{i}\left(\alpha_{\|}, \theta\right)\right]} \\
& \left.\times \prod_{\alpha \in \Delta_{n}^{+}} \frac{1+q^{m-1 / 2} \exp [-\mathrm{i} \phi] \exp \left[-\mathrm{i}\left(\alpha_{\|}, \theta\right)\right]}{1-q^{m} \exp [-\mathrm{i} \phi] \exp \left[-\mathrm{i}\left(\alpha_{\|}, \theta\right)\right]}\right] .
\end{aligned}
$$

For the ghost sector the difference from [1] comes from the bosonic ghosts. Its contribution to the character is

$$
\chi^{\text {gh } \operatorname{Part}_{1}}(q, \theta)=\prod_{m=1}^{\infty}\left[\left(\frac{1}{1+q^{m-1 / 2}}\right)^{2\left(r_{\mathfrak{g}}-1\right)} \prod_{\alpha \in \Delta_{c}}\left(\frac{1}{1+q^{m-1 / 2} \exp [\mathrm{i}(\alpha, \theta)]}\right)^{2}\right] .
$$

The bosonic superconformal ghosts give

$$
\chi^{\text {gh } \text { Part }_{2}}(q)=\prod_{m=1}^{\infty}\left(\frac{1}{1+q^{m-1 / 2}}\right)^{2} .
$$

Putting things together, one ends up with the following expression for the ghost sector of the character

$$
\begin{aligned}
\chi^{g h}(q, \theta) & =\exp \left[2 \mathrm{i}\left(\rho_{\mathfrak{h}^{\prime}}, \theta\right)\right] \prod_{\alpha \in \Delta_{c}^{+}}(1-\exp [-\mathrm{i}(\alpha, \theta)])^{2} \\
& \times \prod_{m=1}^{\infty}\left[\left(\frac{1-q^{m}}{1+q^{m-1 / 2}}\right)^{2 r_{\mathfrak{g}}} \prod_{\alpha \in \Delta_{c}}\left(\frac{1-q^{m} \exp [\mathrm{i}(\alpha, \theta)]}{1+q^{m-1 / 2} \exp [\mathrm{i}(\alpha, \theta)]}\right)^{2}\right]
\end{aligned}
$$


The signature function is determined in the same way and the final result for the ghost sector is

$$
\begin{aligned}
\Sigma^{g h}(q, \theta, \phi) & =\exp \left[2 \mathrm{i}\left(\rho_{\mathfrak{h}^{\prime}}, \theta\right)\right] \prod_{\alpha \in \Delta_{c}^{+}}(1-\exp [-\mathrm{i}(\alpha, \theta)])(1+\exp [-\mathrm{i}(\alpha, \theta)]) \\
& \times \prod_{m=1}^{\infty}\left[\left(\frac{1-q^{m}}{1+q^{m-1 / 2}}\right)^{r_{\mathfrak{g}}}\left(\frac{1+q^{m}}{1-q^{m-1 / 2}}\right)^{r_{\mathfrak{g}}}\right. \\
& \left.\times \prod_{\alpha \in \Delta_{c}}\left(\frac{1-q^{m} \exp [\mathrm{i}(\alpha, \theta)]}{1+q^{m-1 / 2} \exp [\mathrm{i}(\alpha, \theta)]}\right)\left(\frac{1+q^{m} \exp [\mathrm{i}(\alpha, \theta)]}{1-q^{m-1 / 2} \exp [\mathrm{i}(\alpha, \theta)]}\right)\right]
\end{aligned}
$$

Putting eqs. (4.10) and (4.14) together we get

$$
\begin{aligned}
\chi_{\mu}^{1}(q, \phi, \theta) & =q^{\frac{\mathcal{c}_{2}^{\mathfrak{g}}(\mu)}{2 k}} \exp \left[\mathrm{i}(\mu, \Theta)+2 \mathrm{i}\left(\rho_{\mathfrak{h}^{\prime}}, \theta\right)\right] \\
& \times \prod_{\alpha \in \Delta_{c}^{+}}(1-\exp [-\mathrm{i}(\alpha, \theta)]) \prod_{\alpha \in \Delta_{n}^{+}} \frac{1}{1-\exp [-\mathrm{i} \phi] \exp \left[-\mathrm{i}\left(\alpha_{\|}, \theta\right)\right]} \\
& \times \prod_{m=1}^{\infty}\left[\left(\frac{1-q^{m}}{1+q^{m-1 / 2}}\right)^{r_{\mathfrak{g}}} \prod_{\alpha \in \Delta_{c}}\left(\frac{1-q^{m} \exp [\mathrm{i}(\alpha, \theta)]}{1+q^{m-1 / 2} \exp [\mathrm{i}(\alpha, \theta)]}\right)\right. \\
& \times \prod_{\alpha \in \Delta_{n}^{+}} \frac{1+q^{m-1 / 2} \exp [\mathrm{i} \phi] \exp \left[\mathrm{i}\left(\alpha_{\|}, \theta\right)\right]}{1-q^{m} \exp [\mathrm{i} \phi] \exp \left[\mathrm{i}\left(\alpha_{\|}, \theta\right)\right]} \\
& \left.\times \prod_{\alpha \in \Delta_{n}^{+}} \frac{1+q^{m-1 / 2} \exp [-\mathrm{i} \phi] \exp \left[-\mathrm{i}\left(\alpha_{\|}, \theta\right)\right]}{1-q^{m} \exp [-\mathrm{i} \phi] \exp \left[-\mathrm{i}\left(\alpha_{\|}, \theta\right)\right]} .\right]
\end{aligned}
$$

Doing the same for the signature function, i.e. putting eqs. (4.11) and (4.15) together, yields the same expression as in eq. (4.16). Thus, due to Lemma 1 we get unitarity.

Let us now determine the character in the Ramond sector. This problem is a bit harder due to the degeneracy of the vacuum. The states in the relative cohomology are defined in eq. (3.14). There is a degeneracy caused by $\gamma_{0}$ (if $r_{\mathfrak{g}}$ is even), $\gamma_{0}^{\tilde{a},-}$ and $\beta_{0}^{\tilde{a},-}$. If one computes the character and the signature function copying the Neveu-Schwarz case, one will find that they are ill-defined and in the form of an alternating sum or zero times infinity. Therefore, one has to introduce some regulator. Let us show how one can do this in a simpler problem, namely the world-sheet supersymmetric flat case. Let us make a simplification and treat only the reducible Majorana representation of $\mathfrak{s o}(9,1)$. Introduce a charge $N^{\prime} \equiv \sum_{a} \lambda_{0}^{a,-} \lambda_{0}^{a,+}-\gamma_{0} \beta_{0}$ which counts the number of excitations of $\lambda_{0}^{a,-}$ and $\gamma_{0}$. Let us introduce this charge into the trace in the character and signature functions as

$$
\operatorname{Tr}\left[\exp \left[i \varphi N^{\prime}\right] \ldots\right]
$$


where ... indicates BRST-invariant operators9. Computing the character corresponding to the zero mode of the fermions yields

$$
(1+\exp [i \varphi])^{5}
$$

and the part corresponding to the zero mode of the bosonic ghost is

$$
\frac{1}{1+\exp [\mathrm{i} \varphi]} \text {. }
$$

Observe that this result is not BRST-invariant because the charge $N^{\prime}$ is not BRSTinvariant. But we can recover the BRST-invariant piece by taking the limit $\varphi \rightarrow 0$. Thus, the insertion of $\exp \left[i \varphi N^{\prime}\right]$ in the trace acts as a regulator of the character. In the end, the character is well-defined and the result is $2^{4}$, which is the dimension of the sum of the two irreducible spinor representations of $\mathfrak{s o}(8)$ that the Majorana representation consists of. One can do the same for the signature function to get

$$
\underbrace{(1+\exp [\mathrm{i} \varphi])^{4}(1-\exp [\mathrm{i} \varphi])}_{\text {fermionic part }} \underbrace{\frac{1}{1-\exp [\mathrm{i} \varphi]}}_{\text {bosonic ghost part }},
$$

which yields the same expansion as for the character.

Let us now adapt this to the present case. Introduce the charge

$$
N=\sum_{a=1}^{\left[r_{\mathfrak{g}} / 2\right]} \lambda_{0}^{a,-} \lambda_{0}^{a,+}+\sum_{a=1}^{\left[r_{\mathfrak{h}^{\prime}} / 2\right]}\left(\tilde{\lambda}_{0}^{a,-} \tilde{\lambda}_{0}^{a,+}-\gamma_{0}^{a,-} \beta_{0}^{a,+}+\beta_{0}^{a,-} \gamma_{0}^{a,+}\right)-\gamma_{0} \beta_{0} .
$$

This charge will be introduced into the trace in the character and signature functions as $\exp [i \varphi N]$. The character for the $\hat{\mathfrak{g}}$ part due to the fermions is

$$
\begin{aligned}
\chi_{\mu}^{\mathfrak{g} \text { Part }}(q, \phi, \theta, \varphi) & =\exp \left[\mathrm{i}\left(\rho_{\mathfrak{g}}, \Theta\right)\right](1+\exp [\mathrm{i} \varphi])^{\left[r_{\mathfrak{g}} / 2\right]} \\
& \times \prod_{\alpha \in \Delta_{c}^{+}}(1+\exp [-\mathrm{i}(\alpha, \theta)]) \prod_{\alpha \in \Delta_{n}^{+}}\left(1+\exp [-\mathrm{i} \phi] \exp \left[-\mathrm{i}\left(\alpha_{\|}, \theta\right)\right]\right) \\
& \times \prod_{m=1}^{\infty}\left[\left(1+q^{m}\right)^{r_{\mathfrak{g}}} \prod_{\alpha \in \Delta_{c}}\left(1+q^{m} \exp [\mathrm{i}(\alpha, \theta)]\right)\right. \\
& \times \prod_{\alpha \in \Delta_{n}^{+}}\left(1+q^{m} \exp [\mathrm{i} \phi] \exp \left[\mathrm{i}\left(\alpha_{\|}, \theta\right)\right]\right) \\
& \left.\times \prod_{\alpha \in \Delta_{n}^{+}}\left(1+q^{m} \exp [-\mathrm{i} \phi] \exp \left[-\mathrm{i}\left(\alpha_{\|}, \theta\right)\right]\right)\right]
\end{aligned}
$$

\footnotetext{
${ }^{9}$ In this case $L_{0}$ and momentum operators.
} 
Putting this together with the known bosonic contribution yields

$$
\begin{aligned}
\chi_{\mu}^{\mathfrak{g}}(q, \phi, \theta, \varphi) & =q^{\frac{\mathcal{C}_{2}^{\mathfrak{g}}(\mu)}{2 k}} \exp \left[\mathrm{i}\left(\mu+\rho_{\mathfrak{g}}, \Theta\right)\right](1+\exp [\mathrm{i} \varphi])^{\left[r_{\mathfrak{g}} / 2\right]} \\
& \times \prod_{\alpha \in \Delta_{c}^{+}} \frac{1+\exp [-\mathrm{i}(\alpha, \theta)]}{1-\exp [-\mathrm{i}(\alpha, \theta)]} \prod_{\alpha \in \Delta_{n}^{+}} \frac{1+\exp [-\mathrm{i} \phi] \exp \left[-\mathrm{i}\left(\alpha_{\|}, \theta\right)\right]}{1-\exp [-\mathrm{i} \phi] \exp \left[-\mathrm{i}\left(\alpha_{\|}, \theta\right)\right]} \\
& \times \prod_{m=1}^{\infty}\left[\left(\frac{1+q^{m}}{1-q^{m}}\right)^{r_{\mathfrak{g}}} \prod_{\alpha \in \Delta_{c}} \frac{1+q^{m} \exp [\mathrm{i}(\alpha, \theta)]}{1-q^{m} \exp [\mathrm{i}(\alpha, \theta)]}\right. \\
& \times \prod_{\alpha \in \Delta_{n}^{+}} \frac{1+q^{m} \exp [\mathrm{i} \phi] \exp \left[\mathrm{i}\left(\alpha_{\|}, \theta\right)\right]}{1-q^{m} \exp [\mathrm{i} \phi] \exp \left[\mathrm{i}\left(\alpha_{\|}, \theta\right)\right]} \\
& \left.\times \prod_{\alpha \in \Delta_{n}^{+}} \frac{1+q^{m} \exp [-\mathrm{i} \phi] \exp \left[-\mathrm{i}\left(\alpha_{\|}, \theta\right)\right]}{1-q^{m} \exp [-\mathrm{i} \phi] \exp \left[-\mathrm{i}\left(\alpha_{\|}, \theta\right)\right]}\right]
\end{aligned}
$$

The character corresponding to the bosonic coset ghosts is

$$
\begin{aligned}
\chi_{\mu}^{g h \operatorname{Part}_{1}}(q, \theta, \varphi) & =\frac{1}{(1+\exp [\mathrm{i} \varphi])^{2\left[r_{\mathfrak{h}^{\prime} / 2}\right]}} \\
& \times \prod_{\alpha \in \Delta_{c}^{+}} \frac{1}{(1+\exp [-\mathrm{i}(\alpha, \theta)])^{2}} \\
& \times \prod_{m=1}^{\infty}\left[\frac{1}{\left(1+q^{m}\right)^{2\left(r_{\mathfrak{g}}-1\right)}} \prod_{\alpha \in \Delta_{c}} \frac{1}{\left(1+q^{m} \exp [\mathrm{i}(\alpha, \theta)]\right)^{2}}\right],
\end{aligned}
$$

and to the superconformal bosonic ghosts

$$
\chi_{\mu}^{g h \operatorname{Part}_{2}}(q, \varphi)=\frac{1}{(1+\exp [\mathrm{i} \varphi])^{\left[r_{\mathfrak{g}} / 2\right]-\left[r_{\mathfrak{h}^{\prime}} / 2\right]}} \prod_{m=1}^{\infty} \frac{1}{\left(1+q^{m}\right)^{2}},
$$

which together with the known contributions of the fermionic ghosts, yields the character for the full ghost sector

$$
\begin{aligned}
\chi_{\mu}^{g h}(q, \theta, \varphi) & =\frac{1}{(1+\exp [\mathrm{i} \varphi])^{\left[r_{\mathfrak{g}} / 2\right]+\left[r_{\mathfrak{h}^{\prime}} / 2\right]}} \prod_{\alpha \in \Delta_{c}^{+}}\left(\frac{1-\exp [-\mathrm{i}(\alpha, \theta)]}{1+\exp [-\mathrm{i}(\alpha, \theta)]}\right)^{2} \\
& \times \prod_{m=1}^{\infty}\left[\left(\frac{1-q^{m}}{1+q^{m}}\right)^{2 r_{\mathfrak{g}}} \prod_{\alpha \in \Delta_{c}}\left(\frac{1-q^{m} \exp [\mathrm{i}(\alpha, \theta)]}{1+q^{m} \exp [\mathrm{i}(\alpha, \theta)]}\right)^{2}\right] .
\end{aligned}
$$


Putting together eqs. (4.23) and (4.26) one will get the character for the $\hat{\mathfrak{g}}^{-}$and ghost sectors

$$
\begin{aligned}
\chi_{\mu}^{1}(q, \phi, \theta, \varphi) & =q^{\frac{\mathcal{c}_{2}^{\mathfrak{g}}(\mu)}{2 k}} \exp \left[\mathrm{i}\left(\mu+\rho_{\mathfrak{g}}, \Theta\right)\right] \\
& \times \frac{1}{(1+\exp [\mathrm{i} \varphi])^{\left[r_{\mathfrak{h}^{\prime}} / 2\right]}} \prod_{\alpha \in \Delta_{c}^{+}} \frac{1-\exp [-\mathrm{i}(\alpha, \theta)]}{1+\exp [-\mathrm{i}(\alpha, \theta)]} \\
& \times \prod_{\alpha \in \Delta_{n}^{+}} \frac{1+\exp [-\mathrm{i} \phi] \exp \left[-\mathrm{i}\left(\alpha_{\|}, \theta\right)\right]}{1-\exp [-\mathrm{i} \phi] \exp \left[-\mathrm{i}\left(\alpha_{\|}, \theta\right)\right]} \\
& \times \prod_{m=1}^{\infty}\left[\left(\frac{1-q^{m}}{1+q^{m}}\right)^{r_{\mathfrak{g}}} \prod_{\alpha \in \Delta_{c}}\left(\frac{1-q^{m} \exp [\mathrm{i}(\alpha, \theta)]}{1+q^{m} \exp [\mathrm{i}(\alpha, \theta)]}\right)\right. \\
& \times \prod_{\alpha \in \Delta_{n}^{+}} \frac{1+q^{m} \exp [\mathrm{i} \phi] \exp \left[\mathrm{i}\left(\alpha_{\|}, \theta\right)\right]}{1-q^{m} \exp [\mathrm{i} \phi] \exp \left[\mathrm{i}\left(\alpha_{\|}, \theta\right)\right]} \\
& \times \prod_{\alpha \in \Delta_{n}^{+}} \frac{1+q^{m} \exp [-\mathrm{i} \phi] \exp \left[-\mathrm{i}\left(\alpha_{\|}, \theta\right)\right]}{1-q^{m} \exp [-\mathrm{i} \phi] \exp \left[-\mathrm{i}\left(\alpha_{\|}, \theta\right)\right]} .
\end{aligned}
$$

Let us now determine the corresponding signature function. This is done in the same way as for the character. By a straightforward calculation one finds

$$
\begin{aligned}
\Sigma_{\mu}^{\mathfrak{g}}(q, \phi, \theta, \varphi) & =q^{\frac{\mathcal{C}_{2}^{\mathfrak{g}}(\mu)}{2 k}} \exp \left[\mathrm{i}\left(\mu+\rho_{\mathfrak{g}}, \Theta\right)\right](1-\exp [\mathrm{i} \varphi])^{\left[r_{\mathfrak{g}} / 2\right]} \\
& \times \prod_{\alpha \in \Delta_{c}^{+}} \frac{1-\exp [-\mathrm{i}(\alpha, \theta)]}{1+\exp [-\mathrm{i}(\alpha, \theta)]} \prod_{\alpha \in \Delta_{n}^{+}} \frac{1+\exp [-\mathrm{i} \phi] \exp \left[-\mathrm{i}\left(\alpha_{\|}, \theta\right)\right]}{1-\exp [-\mathrm{i} \phi] \exp \left[-\mathrm{i}\left(\alpha_{\|}, \theta\right)\right]} \\
& \times \prod_{m=1}^{\infty}\left[\left(\frac{1-q^{m}}{1+q^{m}}\right)^{r_{\mathfrak{g}}} \prod_{\alpha \in \Delta_{c}} \frac{1-q^{m} \exp [\mathrm{i}(\alpha, \theta)]}{1+q^{m} \exp [\mathrm{i}(\alpha, \theta)]}\right. \\
& \times \prod_{\alpha \in \Delta_{n}^{+}} \frac{1+q^{m} \exp [\mathrm{i} \phi] \exp \left[\mathrm{i}\left(\alpha_{\|}, \theta\right)\right]}{1-q^{m} \exp [\mathrm{i} \phi] \exp \left[\mathrm{i}\left(\alpha_{\|}, \theta\right)\right]} \\
& \left.\times \prod_{\alpha \in \Delta_{n}^{+}} \frac{1+q^{m} \exp [-\mathrm{i} \phi] \exp \left[-\mathrm{i}\left(\alpha_{\|}, \theta\right)\right]}{1-q^{m} \exp [-\mathrm{i} \phi] \exp \left[-\mathrm{i}\left(\alpha_{\|}, \theta\right)\right]}\right]
\end{aligned}
$$

One can proceed in the same way for the ghost sector and end up with the expression

$$
\Sigma_{\mu}^{g h}(q, \theta, \varphi)=\frac{1}{(1+\exp [\mathrm{i} \varphi])^{\left[r_{\mathfrak{h}^{\prime}} / 2\right]}(1-\exp [\mathrm{i} \varphi])^{\left[r_{\mathfrak{g}} / 2\right]}} .
$$


If one puts eqs. (4.28) and (4.29) together determining $\Sigma_{\mu}^{1}(q, \phi, \theta, \varphi)$ one discovers that it is equal to eq. (4.27), even before the limit $\varphi \rightarrow 0$. This implies that if the signature function and the character of the $\tilde{\hat{h}}^{\prime}$ sector are equal, then the full character and signature function are equal. By Lemma 1 this gives unitarity. As this occurs when the representation of $\tilde{\hat{h}}^{\prime}$ is unitary, i.e. when $\hat{\tilde{\mu}}$ is dominant integral, we have now proven the theorem.

Let now consider the converse of the theorem, i.e. that it is necessary that the representation of the auxiliary sector is dominant integral to have unitarity. As remarked in the introduction, the proof presented in [1] is not complete for higher rank theories. For $r_{\mathfrak{g}}=2$ i.e. the cases $S U(2,1) / S U(2)$ and $S L(4, \mathbb{R}) / S U(2)$, however, the proof holds. This gives immediate implications to the present case, as the bosonic sector is part of the supersymmetric model. Thus, we get the following.

Theorem 2 Let $\left(\mathfrak{g}, \mathfrak{h}^{\prime}\right)$ be either $(\mathfrak{s u}(2,1), \mathfrak{s u}(2))$ or $(\mathfrak{s l}(4, \mathbb{R}), \mathfrak{s u}(2)), \hat{\mu}$ be an antidominant weight and assume $\mathcal{H}_{\hat{\mu} \hat{\mu}}^{Q}$ to be non-trivial. If $\tilde{\tilde{\mu}}$ is not a dominant weight then there will always exist a state in $\mathcal{H}_{\hat{\mu} \hat{\mu}}^{Q}$ which is non-unitary.

It is straightforward, using similar expressions as the ones above for the characters and signature functions, to prove unitarity for the supersymmetric coset $G / H$ i.e. when we gauge the full compact subalgebra but not imposing the super Virasoro constraints. This is a generalization of the results for the bosonic case proven in [1]. The fact that we get unitarity for these cases is natural to expect, as we no longer have any time-like excitations. We will present a more detailed report on this and further similar cases in a forthcoming publication.

Table 1:

\begin{tabular}{ccccccccc} 
Coset $\mathfrak{g} ; \mathfrak{h}^{\prime}$ & $\operatorname{dim}(\mathfrak{g})$ & $\operatorname{dim}\left(\mathfrak{h}^{\prime}\right)$ & $g_{\mathfrak{g}}^{\vee}$ & $g_{\mathfrak{h}^{\prime}}^{\vee}$ & $c_{\max }$ & $k_{\max }$ & $c_{\min }$ & $d$ \\
\hline $\mathfrak{s u}(2,1) ; \mathfrak{s u}(2)$ & 8 & 3 & 3 & 2 & 13,5 & -3 & 7.5 & 5 \\
$\mathfrak{s u}(3,1) ; \mathfrak{s u}(3)$ & 15 & 8 & 4 & 3 & 15 & -6 & 10.5 & 7 \\
$\mathfrak{s u}(4,1) ; \mathfrak{s u}(4)$ & 24 & 15 & 5 & 4 & 15 & -40 & 13.5 & 9 \\
$\mathfrak{s u}(2,2) ; \mathfrak{s u}(2) \oplus \mathfrak{s u}(2)$ & 15 & $2 \times 3$ & 4 & 2 & 15 & -32 & 13.5 & 9 \\
$\mathfrak{s o}(3,2) ; \mathfrak{s o}(3)$ & 10 & 3 & 3 & 2 & 15 & -6 & 10.5 & 7 \\
$\mathfrak{s o}(4,2) ; \mathfrak{s o}(4)$ & 15 & 6 & 4 & 2 & 15 & -32 & 13.5 & 9 \\
$\mathfrak{s o}(6) ; \mathfrak{s u}(3)$ & 15 & 8 & 4 & 3 & 15 & -6 & 10.5 & 7 \\
$\mathfrak{s} \mathfrak{p}(4, \mathbb{R}) ; \mathfrak{s u}(2)$ & 10 & 3 & 3 & 2 & 15 & -6 & 10.5 & 7
\end{tabular}

In Table 1 we present the different world-sheet supersymmetric string theories which have a unitary spectrum. As the level of the subalgebra is an integer one can have a largest 
possible value of the conformal anomaly which satisfies $c \leq 15$, as can be seen from eq. (1.3). This value of $c$ is called $c_{\max }$ and the corresponding value of the level is called $k_{\max }$ in table 1. In the table we also give the minimal value of the conformal anomaly which arises in the limit $k \rightarrow-\infty$. Furthermore, we give the number of dimensions of the space-time background, denoted by $d$. Note that one always gets an odd dimension as the space-like degrees of freedom are associated with the noncompact roots, which come in pairs.

In this table there are a few isomorphisms, $\mathfrak{s o}(4,2) \cong \mathfrak{s u}(2,2), \mathfrak{s o}^{*}(6) \cong \mathfrak{s u}(3,1)$, $\mathfrak{s o}(3,2) \cong \mathfrak{s p}(4, \mathbb{R}), \mathfrak{s o}(3) \cong \mathfrak{s u}(2)$ and $\mathfrak{s o}(4) \cong \mathfrak{s u}(2) \oplus \mathfrak{s u}(2)$.

\section{Acknowledgements}

S.H. is partially supported by the Swedish Research Council under project no. 621-20053424 .

\section{References}

[1] J. Björnsson and S. Hwang, Nucl. Phys. B 797 (2008) 464 [arXiv:0710.1050 [hep-th]].

[2] S. Hwang, Phys. Lett. B 435 (1998) 331. [arXiv:hep-th/9806049].

[3] M. Henningson, S. Hwang, P. Roberts and B. Sundborg, Phys. Lett. B 267 (1991) 350.

[4] J. M. Maldacena and H. Ooguri, J. Math. Phys. 42 (2001) 2929 [arXiv:hep-th/0001053].

[5] J. M. Maldacena, H. Ooguri and J. Son, J. Math. Phys. 42, 2961 (2001) [arXiv:hep-th/0005183].

[6] J. M. Maldacena and H. Ooguri, Phys. Rev. D 65, 106006 (2002) [arXiv:hep-th/0111180].

[7] P. Di Vecchia, V. G. Knizhnik, J. L. Petersen and P. Rossi, Nucl. Phys. B 253 (1985) 701.

[8] E. Abdalla and M. C. B. Abdalla, Phys. Lett. B 152 (1985) 59.

[9] P. Goddard, A. Kent and D. I. Olive, Phys. Lett. B 152 (1985) 88.

[10] Y. Kazama and H. Suzuki, Nucl. Phys. B 321 (1989) 232. 
[11] Y. Kazama and H. Suzuki, Phys. Lett. B 216 (1989) 112.

[12] D. Karabali and H. J. Schnitzer, Nucl. Phys. B 329 (1990) 649.

[13] H. Rhedin, Phys. Lett. B 373 (1996) 76 [arXiv:hep-th/9511143].

[14] J. M. Figueroa-O'Farrill and S. Stanciu, [arXiv:hep-th/9511229].

[15] J. Fuchs and C. Schweigert, "Symmetries, Lie Algebras And Representations: A Graduate Course For Physicists," Cambridge, UK: Univ. Pr. (1997) $438 p$

[16] H. Jakobsen, J. Funct. Anal. 52 (1983) 385.

[17] N. N. Shapovalov, Functional Anal. Appl. 6 (1972), 307.

[18] V. G. Kac and D. A. Kazhdan, Adv. Math. 34 (1979) 97.

[19] I. B. Frenkel, H. Garland and G. J. Zuckerman, Proc. Nat. Acad. Sci. 83 (1986) 8442.

[20] S. Hwang and H. Rhedin, Nucl. Phys. B 406 (1993) 165. [arXiv:hep-th/9305174].

[21] S. Hwang and H. Rhedin, Mod. Phys. Lett. A $10 \quad(1995) \quad 823$ [arXiv:hep-th/9408087]. 